\title{
The Quality of Mathematical Education in the Context of Semiotics
}

\author{
Nina Tarasenkova* \\ Bohdan Khmelnytskyy National University at Cherkasy, Cherkasy, Ukraine \\ *Corresponding author: ntaras7@ukr.net
}

Received September 29, 2013; Revised October 12, 2013; Accepted November 03, 2013

\begin{abstract}
The content of mathematics education takes on a specific form of existence in a variety of shells verbal and non-verbal, which are created with the help of sign and symbol means of a different nature. The language is the main means. In teaching signs and symbols perform a substitutional, cognitive, and communicative function; build up an informational basis of students' learning activities. In mastering learning contents signs and symbols are the subject of study, and an instrument of knowledge. Operating sign and symbolic means is a necessary component of students' learning activities, which is directly related to semiosis in students' training activities and enrichment of the conceptual structures possessed and acquired by the students. Mastering different means of fixing the training material contents and every kind of sign and symbolic activity, namely - substitution, coding (decoding), schematization, and modeling is the basis of the information culture and a prerequisite for the development of each student's personality. The selection and use of sign and symbol means should be made on the basis of the analysis of conflicts between the logical and visual. Such conflicts may have not only an objective, historically conditioned character. Most often they are generated by subjective factors - the advent of the students' misunderstanding of the contents of the material and the negative attitudes to the possibility to understand the content, inability to place content in different sign and symbolic shell, the presence of adhesions (but not a dialectical unity) of content and form which were formed in the prior learning students' experience, etc. Adequate conditions for the training and development of all students in the course of mathematics acquisition are created through a comprehensive, systematic and activity approaches to the use of verbal and non-verbal sign and symbol means. Thus, the new goal in teaching mathematics is the ensuring of the two processes unity: the formation of subject knowledge, skills and abilities, on the one hand, and the experience of independent sign and symbolic activities of students, on the other hand.
\end{abstract}

Keywords: teaching mathematics, semiotics, sign and symbol means, conflicts between logical and visual

Cite This Article: Nina Tarasenkova, "The Quality of Mathematical Education in the Context of Semiotics.” American Journal of Educational Research 1, no. 11 (2013): 464-471. doi: 10.12691/education-1-11-2.

\section{Introduction}

The problem of ensuring the quality of mathematics education has always been at the forefront of research in the field of didactics of mathematics. The successful solution of this problem has been not only the scientific community's concern, but also that of school and university teachers and professors of mathematics, and the students themselves. It has many reasons to it as the quality of education, including mathematical education, is the key to successful self-actualization of teachers and teachers and students, to their career development in the future. At the same time, experience shows that traditional approaches and well-known research methods do not allow for a detailed analysis of the reasons impeding the achievement of the objectives of education and ensuring its quality. And dealing with an incomplete spectrum of reasons it is a priori impossible to find a more effective approaches to solving any problem, including those named. Thus, the use of cross-sectoral analysis and synthesis as a scientific method of research is objectively necessary. It has been established [23] that the solution to improving the quality of mathematical education is impossible without the involvement of semiotics and its specific methods of analysis. Research in this field may give new meaning to scientific and methodological research, fill it with new content, and lead to serious theoretical and practical results.

\section{What Semiotics Can Do for the Didactics of Mathematics}

Our research established that it is not only the contents of the subject, its essence and logical organization that are of specific importance in teaching mathematics and pupils' development, but also those forms in which the content is materialized and comes into existence. Understanding abstract mathematical context and operating it is impossible without certain semiotic activities, as content is kept in some shell and its transformation is connected with certain transformations 
of this shell. It is only then when content and form of mathematical abstractions appear before pupils in their dialectical unity, one can speak of conscious mastering the content. The so-called formalism in pupils' knowledge is the manifestation of cohesion of content and form, and this cohesion is the antipode of their dialectical unity.

Under the existence of such cohesions in pupils' personal experience, the formation of a positive egoconception as one of the motive powers in schoolchildren personality formation is next to impossible. The situation is aggravated by the fact that the content of the objects which are to be learned in the school course of mathematics is mono-semantic. Its interpretation or application may be either correct or incorrect - tertium non datur. In this connection, the amount of the pupils' "levels of freedom" in teaching and studying mathematics on quite objective reasons cannot be the same as in studying other school subjects [1]. In particular, it is connected with this or that level of the power of personalization mechanisms manifestation.

In teaching mathematics this power is much smaller in comparison with meaning-oriented (Literature and all art subjects) and positional-oriented subjects (History, Law, native and foreign languages).

Another thing is when a definite mathematical content allows being placed in different shells and pupils learn to operate each of them, to substitute shells without damaging the content and to differentiate the specificity of contents by similar shells, etc. It is here that we see new possibilities of increasing the amount of pupils' "levels of freedom" in studying mathematics and raising the efficiency of the teaching process. It makes up the essence of the principle of maximization of the diversity of pupils' personalities. This principle is new for the theory and methods of teaching mathematics. It brings semiotic approach into education.

\section{The Origins of the Semiotic Approach in Education}

The first steps in developing semiotic approach in education may be traced back already to the period, when main conceptual principles of sign and language approach to human psyche and establishing its specificity as distinct to the psyche of animals were being worked out (E. Cassirer [13], M.M. Bakhtin [2], L.S. Vygotskiy [9]). In accordance with L.S. Vigotskiy's cultural-historical concept in which perception of sign and language approach has changed and this approach has acquired new stuffing, signs (and language signs in particular) and ways of their application are the reasons for the emergence of children's higher psychological functions. These functions originally develop as indirect, cultural, social and specifically human.

E.V. Yilyenkov [12] considers that functional existence of a symbol consists in its being the means of exposing the essence of other things taken in on a perceptional level, that is - exposing of the universal. At the same time, N.G. Salmina [20] emphasizes that in teaching process such functional distinctions of signs and symbols is not essential and they can be viewed in total - as sign and symbol means. O.M. Pyatigorskiy [19] points out that the man lives in "the world of choice", and the choice brings about signness. Signness appears in the process of compressing options and choosing one realization out of multiplicity. So, the procession of information by a human is always accompanied by semiosis.

We find the realization of semiotic approach to mathematical education in such interpretation of the problems of teaching methods in which the stress is laid on the connection of objectives, contents, methods, means and organizational forms of instruction with structure and functioning of sign systems in the course of which pupils' semiosis is correlated with educational process. From this position teaching mathematics should be organized as an aimed process consisting in forming functioning semiotic systems for students.

In methodological research done earlier, only separate sides of the problem of instructing pupils how to use some definite substitutions of mathematical abstractions were under study. In particular, there were considered the problems of teaching pupils the symbolic language of mathematics (G.P. Bevz [3], N.Y. Vilenkin [7], V.G. Kovalenko [14], A.N. Kolmogorov [15], Z.I. Slepkan [21], A.A. Stolyar [22], T.N. Khmara [24] and others), use of some definite means for the mathematization of situations in the process of solving mathematical problems with a plot (A.G. Geistut [10], B.A. Kordemskiy and A.I. Ostrovskiy [16], A.Y. Tsukar [25] and others). The recommendations for teachers on how to prepare and use visual aids in the teaching process were worked out (M.P. Bobrovnik [5], V.G. Boltyanskiy [6], G.A. Vladimirskiy [8] and others).

Still, in the works of our forerunners there has not been found a full spectrum of sign and symbol means which may and should be used as shells of mathematical abstractions which are studied in the school course of mathematics; the specificities of each type of SSM in teaching mathematics have not been exposed; there has been no research into mathematical education and pupils' semiosis in their entity; semiotic aspect of differentiation of the instruction process has not been scrutinized.

\section{The Sign-symbolic Means in Teaching Mathematics}

Semiotics studies the specificity of signs and their systems. Its subject embraces not only the study of the peculiarities of formal structure of sign systems (syntactics), but also the analysis of relations between signs and their contents (semantics), investigation of the processes of creating, understanding and interpreting signs and symbols by man (pragmatics), peculiarities of using signs in designating function (sigmatics), and also - their application in a communicative process (socio-semiotics). it is not by accident that pragmatics domineers in our research.

It's common knowledge that Mathematics as science studies spatial forms and quantitative relations of objects, phenomena and processes of actual world. Detached from the other properties, they altogether create specific for mathematics, idealized thingness, certain abstraction that doesn't exist in reality but reflects it. For a certain abstraction to become an object of analysis it should be fixed by some outer means, that is - it should be materialized. Mathematical science makes use of its 
specific apparatus not only to build its own theory, but to fix the created abstraction from without. For this different signs and symbols are used and, after N.G. Salmina [20], in the aggregate, we call them sign and symbolic means (SSM).

\subsection{The Functions of SSM}

In the process of studying mathematics SSM perform substitution, cognitive and communicative functions. Abstract mathematical objects which are materialized in a certain way (here the substitution function of SSM is realized) become fit for pupils' perception and turn into perceptive material which is specific for/in mathematics. In cognitive processes this material is used to single out both - essential (on perceptive level) and concrete moments in actions which transform materialized abstract objects and produce knowledge and ways of its application (here the cognitive function of SSM is realized). Communicative function of SSM in teaching is aimed at insuring the transmission of the message - from teacher to pupil and back, from one pupil to another, and from socium to a pupil through different carriers of information.

\subsection{The Groups of SSM}

We have established that verbal and non-verbal means should be regarded as separate groups of SSM in teaching mathematics.

To make the organization of mathematical instruction didactically balanced, one should know not only semiotic peculiarities of SSM mentioned above, but also - how evolutional semiotic lines may be arranged, how they should be organized according to the age peculiarities of the pupils and how these lines are to be introduced in class.

\subsubsection{The Verbal SSM}

Among verbal means it is rational to differentiate such SSM as: object texts, terminology, texts of mathematical problems, texts of questions, pictograms (or texts with the elements of pictography).

To language sign-symbolic means used in teaching Maths we refer:

- object texts, such as: formulations of concepts definitions and of mathematical facts (axioms, theorems, and formulas), types of mathematical operations (algorithms, rules, heuristic schemes, etc.), or descriptions that replace the strict wording of the object;

- terminology of Mathematics;

- logical-mathematical symbols used to denote isolated objects in mathematics;

- content and analytical interpretation of mathematical concepts, facts and operations which acquire the forms of formalized mathematical sentences expressions, equations, inequalities, identities, etc.;

- educational texts with recorded contents of some local knowledge of the theory of Mathematics;

- mathematical problems phrasing;

- question phrasing;

- pictography or records with pictographic elements.

Now we shall specify the features of term words as one of the varieties of SSM, used in teaching mathematics.
In Mathematics there can be used two types of terms nominative (French - nominatif - one that serves naming objects, phenomena, qualities, actions, etc.) and auxiliary terms. Each typological group of terms can be divided into certain classes.

Thus, in the nominative mathematical terminology we can differentiate two classes. The first one should be called a general class of nominative terms. It includes: 1 ) the names of mathematical objects (for example, the terms "a straight line", "plane", "number", "equation", "multiplier", etc.), 2) the names of mathematical operations (for example, the terms "add", "exponentiation", "sine", "module", etc.), and 3) the names of mathematical relationships (for example, the terms "equals", "more", "less", "belongs to", "parallel", etc.); 4) general names of objects of assimilation (e.g, the terms "concept", "theorem", "property", "rule", "method", etc.), 5) common names of sign and symbolic reification of mathematical contents, such as: names of object texts (for example, the term "definition", "formulation", "description", etc.), the names of other language SSM (for example, the terms "signs of arithmetic operations", "mathematical symbols", "nomenclature", "icon", etc.); nonlinguistic SSM name (for example, the terms "figure", "layout", "table", "schedule", "chart", etc.), 6) umbrella names of the object texts semantic components (for example, the terms "a signified concept", "condition (premise)", "claim (conclusion)", "proof", "example", etc.), 7) signifying words and phrases (from Lat. significo - I give a sign, signal) used as differentiating features, dividing the detailed text into its semantic components: cause from consequence, condition from evidence, thesis and arguments, etc. (e.g: "if ... then...", "given, prove, proof", etc.

To the second class of the mathematics nominative terminology we refer individual nominative terms. They reflect the names of specific objects and are formed by the specification of the relevant general terms. For example, the individual nominative terms include the following names: "the notion (of what?) of the root of the equation", "Pythagorean (whose?) Theorem", "vertical angles (what kind of?) theorem", "formulation (of what?) of the basic properties of fractions", quadratic (what kind of?) equation, etc.

Individual nominative terms are subject to the requirements of maximum scientific accuracy and invariance - regardless of the context.

In mathematics there are also terms whose sign and symbolic shell has the form of a nominative term with the specification, but in their content they do not fix the relationship between generic and species concepts. These are the terms "decimal", "cut pyramid" and others.

We fully share G.Bevz's opinion [4,9] that these terms should be considered indecomposable terms.

The need for consideration of auxiliary terms is dictated by the characteristics of mastering the mathematics nominative terminology and its proper use. Auxiliary terms may serve as an additional explanation, clarification, comparison, or a semantic mnemonic guide, instructions, etc.

\subsubsection{The Non-verbal SSM}

Among non-verbal means one should single out the pictures of geometrical figures, meaningful graphical 
interpretations of mathematical notions and facts, charts, diagrams, schemes, graphs, analytical configurations, objects of reality, models and constructions, graphic illustrations, means of plastic art.

In teaching mathematics the class of non-linguistic sign and symbol means is represented by the following SSM

- images of geometric shapes, which may be used as a visually plausible and visually distorted images, illustrations of different types (two-dimensional spatial iconic SSM );

- layouts and designs that in simulated situations will pass for modeled objects, phenomena and events of the real world ( three-dimensional spatial iconic SSM );

- tables, charts, diagrams, schematic graphics as a means of mapping structured entities (arbitrary twodimensional spatial SSM );

- real items used in substitution functions (arbitrary three-dimensional spatial SSM );

- content and graphic interpretation of mathematical concepts, facts and ways of activity rate ( intrinsic iconic SSM );

- natural language texts and mathematical sentences specifically placed on the plane (analytical configurations), content, visual and content and visual accents (intrinsic arbitrary SSM);

- plastic display of the essence of mathematical concepts, facts, or ways of life, such as the rules of conventional division of fractions, monotonic functions, perpendicular lines, dramatization, the game (human actions as iconic SSM);

- non-verbal communication, rituals (human action as arbitrary SSM).

However, the non-verbal semantic-symbolic means should be considered also in terms of their didactic purpose in learning mathematics. Some of them are the SSM which are the objects of learning. These are - images of geometric shapes; content and graphic interpretation of concepts, facts and ways of life taught in math courses, tables, charts, diagrams, and schematic graphics. The second group includes those SSM that mostly perform an accompanying function in the learning process. These are the rest of the above-mentioned non-verbal SSM including educational illustrations. In this group some reorganization should be undertaken. Particularly expedient it is to view three-dimensional spatial SSM in their unity (real objects, models, and designs), as in the learning process they are used in the functional unity.

In our opinion, there shouldn't be any separation from each other such SSM species as human actions. A united group of these techniques is called plastics. In their turn, plastic techniques of fixing mathematical contents should be examined together with such representatives of iconic two-dimensional spatial SSM as illustrations. One of the arguments in this regard incurs from the fact that the images contained in textbooks and manuals on mathematics, often show the contents and sequence of certain human actions.

\subsection{The Activity with SSM}

Application and transformation of sign and symbol means is a rather specific activity and it is called sign and symbol activity (SSA).
SSA comprises substitution, encoding and decoding, schematization and modeling. It has been established that in teaching mathematics each type of SSA should be divided into subtypes [23]. This brings about a complex of problems waiting for their solution.

Pupils' ability to master any SSA lies in the original capability of a human psyche to develop. In its turn, the realization of such ability, forming wholesome SSA through instruction is one of many factors of further development of a pupil's psyche and its symbolic function.

This function is an abstract ability to separate content and the form of its expression, define the type of their connection, analyze content through its sign and symbol form, operate and transform SSM (L.S. Vigotskiy [9], J. Piaget [17], G.S. Kostyuk [18] and others).

\subsubsection{The Activity of Substitution}

The activity of substitution ought to be approached in its broad and narrow meaning. Speaking about broad meaning we have in mind the use of substitutes in other types of SSA (e.g. - the picture of a directed segment is used when introducing the notion "vector", for demonstrating the connection between two objects and as a model of the movement direction). Narrow meaning deals with the use of a substitute instead of the substituted phenomenon (e.g.: a note $3+2=5$ as functional reflection of the sense of activities in counting the books on bookshelves). And here it is of importance to analyze how the process of creating sign and symbol shells by pupils (semiosis) is connected with a teacher's sign and symbol activity (or the one of the author of the textbook in which the results of this activity were presented).

\subsubsection{The Activity of Encoding}

The activity of encoding which consists in translating the reality (or the text which describes reality) into the language of some sign system should be examined separately according to designated and situational purposes. The first purpose of encoding is realized in forming in pupils the knowledge in the form of code structures (according to "shell - nucleus - operator" principal scheme and on condition that the constructs "positive - negative" are formed). Encoding, according to its situational purpose, implies the use of terminological, symbolical and word and graphical code of a notion, fact or mode of activity.

Methods of forming knowledge as a set of code structures, peculiarities of the organization (in the teaching process) of the encoding activity with its situational purpose in mind may become subjects for further research.

\subsubsection{The Activity of Decoding}

Decoding is connected with extracting content from a given sign and symbol shell. In mathematical education we connect this type of SSA with reading (decoding some data in the text created with the native language means), deciphering (decoding data in the text created by means of the formalized language of mathematics and pictography); identification and recognition (of geometrical figures, graphs of functions, etc.), decoding other non-verbal data (with or without verbal support). It is the field where the research of pragmatically aspect of semiotics is most important. 


\subsubsection{The Activity of Re-coding}

In re-coding activity (which is another variety of SSA) the transition from one shell of content to its other shell is realized. The identification of the content by its shell takes place at the stage of entrance decoding (e.g. the given equation is recognized as a quadratic one). At the second stage of re-coding there takes place the transformation of the content in accordance with the logics of the object of activity (e.g. - evaluating the roots of the quadratic equation by formula of roots). At the stage of final encoding we observe the turn of the updated content into a new sign and symbol shell (e.g. - presentation of the originally given quadratic equation as a nought equal product). Here there also many issues to be researched, in particular - the problem of the connection "pupil - sign meaning” and vice versa.

\subsubsection{The Activity of Schematization}

Learning with the support of some scheme which reflects reality through verbal and non-verbal means is realized in schematization activity. At the same time two situations are completely different in their essence. In the first situation the schemes which are known to the pupils (as these schemes were being mastered) are used. They are used when the new material is introduced; in the process of solving problems with the support of some algorithm or heuristic scheme; in the process of generalizing and systematizing. We connect the second situation with the use of just created schemes - verbal, non-verbal and phantom ones. Most often such schemes appear when the method of rational problems is used and as a result of didactically reasoned structuring the system of questions and tasks. Each situation becomes inimitable depending on the subject matter, SSM which are used in every particular case, the skills level and the level of learning ability. It is another point which is still to be thoroughly studied.

\subsubsection{The Activity of Modeling}

Many scientific papers have been written on the content aspect of the modeling activity, but the semiotic aspect of this needs further consideration.

Modeling is such SSM-using activity that aims at obtaining new information about the objective reality through the operation by these techniques. In students' learning activity the study of mathematics modeling has certain peculiarities. Their detailed analysis allowed us of identifying some types of activities.

The first type of modeling is associated with the mathematization of situations - the creation of mathematical models that allow us of studying reality by means of mathematicians (perhaps through the shell of the texts formed by verbal means and those that describe this reality). SSM-using activities of this type are commonly referred to as mathematical modeling. Mathematical equations play the role of the models that are built in the process of mathematical simulation, equations, inequalities and their systems, etc., or non-verbal sign and symbol means - pictures, charts, graphs, tables, etc.

Simulation of the second type differs from the previous one. Its characteristic feature is that it is carried out entirely in entirely conditional terms. In reality act as Such sign and symbolic objects: as equations, inequalities or their system that are to be explored, solved, etc., perform the role of the reality. Contact with reality during this simulation is too remote.

For example, in the study of a certain equation, and especially when the substitution of the variables is used, human consciousness does not at once fix the fact that this equation itself is already a model of reality, in particular, everyday, industrial, etc. The essence of this reality also stays outside the minds of students. There is an urgent need of new information on this equation, rather than of finding out the information about the real situation, which it simulates. In fact, in the study of the equation the construction of a new model of semantic- symbolic reality which is represented by this equation takes place. In other words, the meta-model is built.

Such modeling should be called meta-modeling. It is closely connected with activities associated with conversion, but is not limited to them. In recoding there takes place the converting of sign and symbolic component of a substitute though its semantic component stays intact. In meta-modeling, like in modeling itself, both - sign and symbolic as well as semantic components of a substitute are altered; in the result of such an alteration a fundamentally new information about the original object of study appears. The detection of this new data is an immediate goal of meta-modeling activity.

However, in the study of mathematics the situations in which the reality research begins as simulation in its true sense - as mathematical modeling, continues as metamodeling and is completed by the results interpretation in terms of the original reality. In other words, metamodeling acts as an inner structural component of mathematical modeling. In this case, the SSM-employing activities should be called composite modeling.

Simulation of this nature is performed when the mathematical model, which is obtained in the course of the mathematization of reality, requires additional study.

Such situations often arise in the study of new educational material when the educational process is based on the definite inductive method, in the process of solving research problems, while solving story problems in which modeling is based on building a parametric model, etc.

In the process of teaching mathematics students should be introduced into the peculiarities of every type of simulation, taught how to differentiate various simulation situations and perform such simulation activities according to the semiotic logics of this activity.

\section{Dialectical Unity of Logical and Visual and Its Role in Teaching Mathematics}

SSM are kind of contents shell, their sign and symbolic forms.

In teaching they can be used in different modalities visual, auditory, motor, tactile etc. However, visual modality is a domineering one. In books, textbooks, manuals and other printed maths education media the contents are fixed in this very modality. Psychologists say that all learning is based on a number of visuals, because for the most part, the process of the contents perception and its initial processing starts with visual recognition. 
Based on the dialectics of relationship between contents and form (Hegel), we found out the nature and role of dialectical logic and visual unity in the mathematical preparation of students the basic theory of conflict between logical and visual is constructed.

\subsection{The Essence of the Dialectical Unity of Logical and Visual}

In accordance with the general laws of learning, the analysis of the reality is made through the analysis of its sign and symbolic form. Herewith, human activity has double determination [26]. It is defined as the internal logic of the subject, and the logic of activity with SSM, which means the knowledge [20].

In the context of these regulations, the contents of the objects of mathematics acquisition should be appropriately called logical (semantic and meaningful); and sign and symbolic content shell filed in the visual modality, visual. Accordingly, the process of visual recognition should be called visual analysis and the content recognition process might be called an analysis of the logical (meaningful) or semantic analysis. Content analysis is a combination of two processes - visual analysis and semantic analysis. By temporal characteristics visual analysis comes before analysis. Sometimes they occur simultaneously.

Logical and visual in learning maths should serve as a dialectical unity. The objective nature of this unity is that being relatively independent, the content and form of its fixation are interrelated, the form is included in the contents, is a part of it and refers to it as part of a whole [11]. This means that the sign and symbolic shell reflects both - internal (intrinsic) features of contents and their external manifestations, moreover, its content and sign and symbolic forms are adequately identical. The realization of these positions is an attribute of modern scientific mathematical theories and projections in the didactics of mathematics.

However, objective dialectical relationships of logical and visual do not mean that these links automatically appear to be a dialectical unity for students as the subjects of education. In teaching math a teacher should work hard to make sign and symbolic means of fixing the school mathematics contents meaningful for students. Only through the teacher's targeted training and hard work combining logical and visual as a dialectical unity can complement personal experience of students. If such unity exists, the application of knowledge by the students shows that the results of the visual analysis do not contradict the results of the semantic analysis, together they reflect the nature of the phenomena being analyzed, any transformations of sign and symbolic shells do not damage the corresponding meaning; proper manipulation of contents is mostly exercised through the manipulations with collapsed and expanded forms and even without full explanation of the meaning by means of natural language.

\subsection{The Notion of Conflict between Logical and Visual}

Dialectical logic and visual unity does not exclude the occurrence of conflicts (contradictions) between them, but rather foresees them.
Objectively, the conflict between the logical and the visual is the general law of contradiction between contents and form. In teaching mathematics its manifestations can be seen rather vividly, for example, while introducing lettering the numbers as a generalized form of their writing and fixation, when classifying triangles by the relation of equality of arms, when new content objectively requires the introduction of new terms - versatile, isosceles and equilateral triangles; while familiarizing students with the concept of irrational numbers as new numerical object whose appearance is dictated by the need to expand the concept of number, and so on.

The subjectivity of the conflict between logical and visual is associated with the specific perception and processing of content by students, with the processes of learning and applying mathematical knowledge and skills. Moreover, subjective conflicts may arise even in those cases when there is no objective basis.

One of the first subjective factors of conflict between logical and visual arising from the lack of students' understanding of the learning mathematical contents introduced for the first time. This means that the chosen sign and symbolic content shell, being objectively adequate, subjectively is not perceived as such. We can say that in this case the semantic-symbolic shell becomes transparent for students - internal, intrinsic features of the content remain invisible to them, and the results of visual analysis do not coincide or conflict with the results of the semantic analysis.

Problems of initial misunderstanding generate such negative phenomenon as the formation of original content and form adhesions. They arise in the personal experience of students as a result of formal learning and manifest that in the future students will not be able to transform the semantic - symbolic shell without damaging the contents; will be unable to detect even content changes if minor changes in the cover occur; standard situations they conduct an incomplete semantic analysis that leads to errors. Under these conditions, a distant transfer of knowledge is out of the question. Thus, any adhesion of shape and form is the antithesis of dialectical combination. The formation of such adhesions in the personal experience of students should be prevented by all means.

Other factors of subjective contradictions between logical and visual in teaching mathematics are associated with encoding and decoding procedures which are necessary in the students' application of knowledge.

\subsection{Hierarchy of Conflicts between Logical and Visual}

In the hierarchy of conflicts between logical and visual one can differentiate between two types of conflicts and their variations within each type. The first type is represented by objective conflicts, arising in the moment of coding new mathematical content by the students.

The second type is represented by those conflicts that are not objectively conditioned. They can appear during decoding the contents (and their variety is as large as large is the variety of different SSM) as well as during their recoding.

\subsubsection{Outline of Division into Conflict Types in Recoding}


Generally, in the group of conflicts it is theoretically possible to identify as many of their species as there are many pairs which will form different SSM used in teaching mathematics. A more precise classification of semantic conflicts can be obtained by sequential division of the basics that are displayed in the following diagram:

1) initial and final sign and symbolic shells are the same name SSM:

a) the transition from verbal to verbal SSM (from expanded form to another expanded form, from expanded form to a folded) form, from folded form to another folded form, from the folded form to expanded form) is made;

b) the transition from non-verbal to non-verbal SSM (from folded form to folded form which is a representative of the same kind of non-verbal SSM, from folded form to folded form that represents another type of non-verbal SSM) is made;

2) the initial and final sign and symbolic environments are opposite SSM:

a) the transition from verbal to nonverbal SSM (from expanded form to a non-verbal language form, from folded language form to non-verbal forms) is made; b) the transition from non-verbal to verbal SSM (from non-verbal form to folded verbal form, from a non-verbal form to a verbal expanded form) is made.

Taking into account the fact that recoding in teaching mathematics can be done in two ways - as a formal sign and symbolic replacement of the shell, which does not involve changes in the content and as a replacement, resulting from the semantic reorganization, then the branching of the classification will increase. And at least one type of conflicts should be divided into two sub-types.

\subsubsection{Types of Recoding Conflicts Observed in Teaching Mathematics}

The analysis showed that some theoretically possible conflicts of this type of learning appear simultaneously in the process of learning with similar manifestations, they are minimized only as a whole. So it is appropriate to combine them by the functional index - the place and the role they perform in teaching and learning.

Therefore, among the recoding conflicts we should distinguish ten types.

1. Conflicts arising during the conversion of object texts (definitions of concepts, definitions of theorems, rules, etc.) into another detailed form built by means of natural language (e.g. the categorical formulation of the theorem is converted into an implicit form.

2. Conflicts arising from recoding the content of the expanded language form into a particular folded verbal form, and vice versa (e.g. during the correlation of the definitions with scientific terms and concepts, the term with the logic- mathematical sign (symbol) etc.).

3-4. The third type conflicts occur during the conversion of the expanded verbal material into a spacegraphical form. The fourth type conflicts are associated with the opposite activities. These types of conflicts arise when students are to relate, for example, the definition of geometric concepts and the corresponding geometrical images, conditions of mathematical problems with the chart (a table, a graph) in which the link between data and unknown quantities is reflected, etc.

5. Conflicts that arise when converting mathematical expressions, equations, inequalities, etc. If the experience of students formed logical and visual adhesions, the occurrence of this type of conflict is inevitable. Experiential learning practice shows that the degree of conflict situations can be significantly lower under the conditions of building visually adequate series, particularly positioned by registration records.

6. Conflicts of this kind are analogous to certain conflicts of the previous group, as they are also associated with leveraging the same name SSM - namely, in content and graphic interpretations. However, despite the relatively high conflict relationships between logical and visual of the sixth type, their solution is a less complex problem in teaching mathematics students than overcome the fifth type conflicts. The fact is that in content and graphic interpretations it is iconic SSM which are mostly used; and the iconoclasm of the sign (symbol) makes it to be the most motivated among all SSM. In addition, operating iconic SSM can occur outside the verbalization of relevant meanings, and thus it is to be exercised through visual thinking in one's super-consciousness.

7. Conflicts related to the procedure of recoding of content and analytical mathematical interpretation of mathematical notion or fact into their graphic (or content and graphical) interpretation or vice versa, when handling relevant content requires the simultaneous use of both interpretations. Most of the conflicts of this type occur when students solve story problems and geometric problems.

8. Conflicts related to the flaws of the so called spatial vision and manifested during the conversion of twodimensional geometrical configuration into the image of spatial figures. Such conflicts get more serious when students have to operate with unlimited stereo-metric objects - directs and planes in space.

9. Conflicts that arise in the process of solving stereometric problems when there is a need for performing one or more of planimetric steps. The essence of these conflicts is associated with the need of decoding the planimetric data on the visually distorted fragments of stereo-metric images and recoding during the transition from visually plausible images to visually distorted ones and vice versa.

10. Conflicts arising in connection with the use of real objects as substitutes when the real physical properties of these objects, their social purpose, and attitudes introduce some communicative noise in the process of learning and in identifying essential and non-essential properties of the ideal object.

In general, the leveling of conflicts between logical and visual allows us of creating favorable conditions for full cognition by the students of the nature of mathematical content, effective exercise of individual subject performance, and handling semantic- symbolic means.

\section{Conclusion}

In conclusion we should admit that the solution of the registered problems will make it possible to conduct a more thorough analysis of mistakes and difficulties faced by the pupils in studying mathematics, to discover their essence and the causes of their appearance, to find theoretically substantiated ways of their elimination and prevention. Finally, this will allow to make instruction 
more effective, create favorable conditions for pupils and to form their personalities purposefully.

All this can and should become the object of further research.

\section{References}

[1] Alekseev, NA, Pedagogical bases of designing a student-centered learning: dissertation, Tyumen State University, Tyumen, 1997, 42.

[2] Bakhtin, M. M, The aesthetics of verbal creativity, Iskusstvo, Moscow, 1986, 445.

[3] Bevz, G. P, Methods of Teaching of Mathematics, Vyshcha shkola, Kyiv, 1989, 367.

[4] Bevz, H.P, "Of numbers", Mathematics in School, № 1, 6-9, 2002, № 2, 23, 2002.

[5] Bobrovnyk, M. P, Zhurbas, M. O, Visual aids in mathematics, Radyans'ka shkola, Kyiv, 1968, 180.

[6] Boltyanskii, V.G, "Functions of educational tools and organization of the search for tasks solutions," Sovetskaya pedagogika, № 10, 40-47, 1975.

[7] Vilenkin, N. Ya, Shvartsburd, S. I, "Statements, expressions, variables", Matematika v shkole, № 3, 34-41, 1970.

[8] Vladimirsky, G. A, Stereoscopic drawings of the geometry: The Album, Uchpedgiz, Moscow, 1963, 176.

[9] Vygotsky, LS, Collected Works, in 6 volumes, Volume 2: Thought and Language, Pedagogika, Moscow, 1982, 504, Volume 3: Problems of development of the mind, Pedagogy, Moscow, 1982, 367.

[10] Geistut, A. G, Khmara, T. M, "Statistics in Pictures", Matematyka v shkoli, № 1, 29-30, 1999.

[11] Hegel, G. F, Science of Logic: In three volumes, Volume 1: The Doctrine of Being, Mysl', Moscow, 1970-1972, 501, Volume 2: The doctrine of the essence, Thought, Moscow, 1970-1972, 248,
Volume 3: The teaching of the concept, Thought, Moscow, 19701972, 374.

[12] Ilyenkov, E.V, Dialectical logic: Essays on the History and Theory, Politizdat, Moscow, 1984, 320.

[13] Cassirer, E. Shortlist. Essay on Man, Gardarica, Moscow, 1998, 784.

[14] Kovalenko, V. G, Sledzinskyy, J. F, Mathematical symbols: A Guide for teachers' self-education, Radyans'ka shkola, Kyiv, 1981, 80.

[15] Kolmogorov, A. N, Mathematics - science and profession, Nauka, Moscow, 1988, 288.

[16] Ostrovsky, A. I, Kordemsky, B. A, Geometry helps Arithmetic, Fizmatgiz, Moscow, 1960, 168.

[17] Piaget, J. Psychogenesis knowledge and its epistemological significance, Semiotics: An Anthology, Academics project, Moscow, Delovaya kniga, Yekaterinburg, 2001, 98-110.

[18] Psychology: Tutorial for pedagogical universities / edited by G.S. Kostiuk, Radyans'ka shkola, Kyiv, 1968, 572.

[19] Piatigorsky, A. M, Some general comments on the review of the text as a kind of signal, School "Languages of Russian culture", Moscow, 1996, 590

[20] Salmina, N. C, The sign and symbol in teaching, Moscow State University Press, Moscow, 1988, 286.

[21] Slepkan, Z. I, Psychological and pedagogical bases of learning mathematics, Radyans'ka shkola, Kyiv, 1983, 192.

[22] Stolyar A. A, Pedagogy of Mathematics, Vysheyshaya shkola, Minsk, 1974, 382.

[23] Tarasenkova, N. A, Using sign and symbolic means in teaching mathematics: Monograph, "Vidlunnya-Plyus", Cherkasy, 2002, 400.

[24] Khmara, T. M, Teaching students the mathematical language, Radyans'ka shkola, Kyiv, 1985, 95.

[25] Tsukar, A.Ya. "Schematization and modeling in solving text problems", Matematika v shkole, № 5, 48-54, 1998.

[26] Yudin, E.G. et al, Science and the world of man, Znaniye, Moscow, 1978, 64. 